\title{
Minireviews
}

\section{Hepatic Bile Formation: Canalicular Osmolarity and Paracellular and Transcellular Water Flow}

\author{
Raul A. Marinelli, Mary Vore, and Norman B. Javitt \\ Instituto de Fisiologia Experimental, Consejo Nacional de Investigaciones Científicas y Técnicas, Facultad de Ciencias \\ Bioquímicas y Farmacéuticas, Universidad Nacional de Rosario, Rosario, Santa Fe, Argentina (R.A.M.); Department of \\ Toxicology and Cancer Biology, College of Medicine, University of Kentucky, Lexington, Kentucky (M.V.); and Department of \\ Medicine and Pediatrics, New York University School of Medicine, New York, New York (N.B.J.)
}

Received August 8, 2019; accepted September 25, 2019

\begin{abstract}
The purpose of this minireview is to show that a new paradigm is developing regarding hepatic bile flow. The focus thus far has been on carrier-mediated transport of bile acids and other solutes, such as glutathione, which create an osmotic gradient for the transcellular and paracellular flow of water into canaliculi. In addition to the physicochemical properties of bile acids, which govern the osmotic gradient, data now exist showing that the tight junctions governing paracellular water flow and Aquaporin-8 water channels governing transcellular water flow are regulated independently. Thus, the rate of water flow into the canaliculus in response to bile acid transport is variable and determines canalicular bile acid concentration, which affects the production and solubilization of cholesterol-lecithin vesicles.
\end{abstract}

\section{Introduction}

Claudin-2 is one of the proteins comprising the tight junctional apparatus that regulates paracellular water flow (Tanaka et al., 2017). Therefore, we can pinpoint the reduction in hepatic bile flow found in the Claudin-2 null mouse (Matsumoto et al., 2014) to events occurring in the canaliculus.

A reduction in transcellular canalicular water flow by estradiol-17 $\beta$-D-glucuronide $\left(\mathrm{E}_{2} 17 \mathrm{G}\right)$ can also be pinpointed to the canaliculus because of a related increase in bile acid concentration. The focus on transcellular water flow relates to the effect of $\mathrm{E}_{2} 17 \mathrm{G}$ on the expression of Aquaporin-8 identified in the canalicular membrane (Huebert et al., 2002). The increase in the concentration of bile acids in the canaliculus as a result of decreased paracellular and/or transcellular

This work was supported by the Agencia Nacional de Promoción Científica y Tecnológica [Grant PICT 2015-0439 (to R.A.M.)].

https://doi.org/10.1124/jpet.119.261115.
These new considerations modify thinking regarding the occurrence of cholestasis and its progression and reorient the design of experimental studies that can distinguish the different determinants of bile flow.

\section{SIGNIFICANCE STATEMENT}

The paradigm that water flow into the canaliculus is determined only by the rate of carrier-mediated transport has been challenged recently by the changes that occur in hepatic bile composition in the Claudin-2 knockout mouse and with the cholestatic effect of estradiol $17 \beta$-D-glucuronide. Thus, a respective reduction in paracellular or transcellular canalicular water flow, probably via Aquaporin 8 , has no significant effect on bile acid excretion. water flow affects their osmolarity and the solubilization of cholesterol and lecithin.

Accepting that water flow into the canaliculus is osmotically determined, as proposed in the 1950s (Sperber, 1959), we need to integrate this concept with these determinants of the rate of paracellular and transcellular water entry, which appear to be independently regulated by the expression of the claudin-2 and Aquaporin-8 proteins. The concept that paracellular and transcellular water flow, although governed by osmotic gradients, is nevertheless independently regulated broadens our approach to understanding the pathogenesis of cholestasis, particularly regarding the effects of xenobiotics.

\section{Determinants of Canalicular Water Flow}

Claudin-2. Claudin-2 null (Cldn $2^{-/-}$) mice are born in the expected Mendelian ratios. Their growth rate, appearance, activity, and behavior are normal (Matsumoto et al., 2014) in contrast to other claudin null models that cause early lethality (Muto et al., 2010; Tsukita et al., 2019). Therefore, this model 
is amenable to many long-term studies. Hepatic bile flow in the claudin- 2 null mouse is $50 \%$ less than in the wild-type animal, but bile acid, bilirubin, lecithin, and cholesterol output are not significantly different from normal, each having an approximately 2-fold increase in concentration (Matsumoto et al., 2014). There is no increase in hepatic or plasma bile acid concentration. The challenge is to understand why water flow can vary in response to a solute load, generated mostly by bile acids.

Aquaporin-8. Aquaporin-8 belongs to a family of transmembrane proteins with the primary role of conducting osmotically driven water molecules across cell membranes (Calamita et al., 2018) (Fig. 1). Aquaporin-8 pore structure and substrate specificity differ substantially from other human aquaporin water channels (Horner and Pohl, 2018). The single water permeability ( $\mathrm{p} f$ ) of Aquaporin-8 expressed in hepatocyte canalicular membranes is approximately 100 -fold less than Aquaporin-1 found in the nephron and ductular regions of the biliary tree and is much closer to Aquaporin0 found in the lens of the eye, which has the lowest permeability coefficient (Horner and Pohl, 2018). The biologic significance of these differences is an area of active investigation. The low Aquaporin-8 intrinsic water permeability likely contributes to the fact that the canalicular membrane has lower osmotic water permeability than the basolateral membrane, and thus it is rate limiting for the osmotically driven transcellular water transport in hepatocytes (Marinelli et al., 2003). In addition, it is reasonable to think that the low permeability of Aquaporin-8 favors relatively more rapid bile acid transport than water flow, thus accounting for micellar concentrations of bile acids in the canaliculus that are necessary for the solubilization of the water-insoluble cholesterol-lecithin vesicles (Crawford et al., 1995).

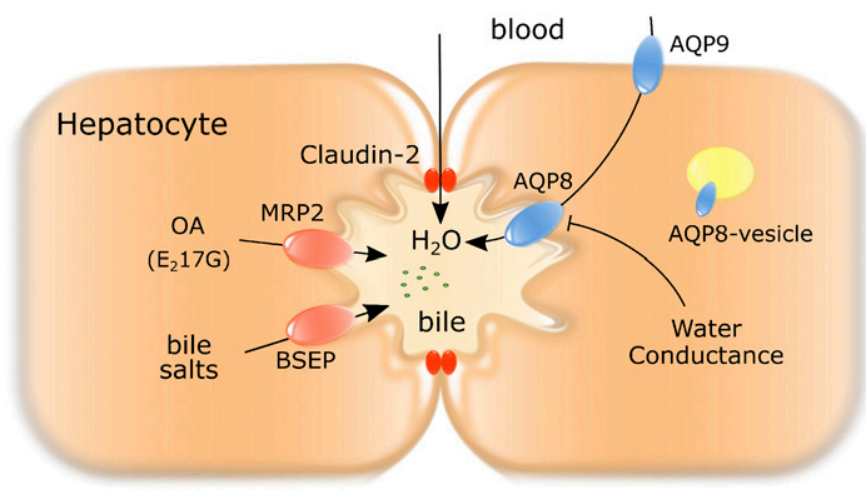

Fig. 1. Hepatocyte canalicular water transport. The output of bile salts via BSEP/ABCB11 generates an osmotic gradient for water movement transcellularly via Aquaporin-9 (AQP9) at the basolateral surface and Aquaporin-8 (AQP8) in the canalicular membrane and paracellularly through claudin-based tight junctions. $\mathrm{E}_{2} 17 \mathrm{G}$ binds to MRP2/ABCC2 and is transported into the canaliculus. After low-dose intravenous injection, canalicular water flow rapidly decreases and bile acid concentration increases (see Fig. 2). Canalicular water permeability is modulated by changes in the number of AQP8 molecules by trafficking and insertion of AQP8-containing vesicles. $\mathrm{E}_{2} 17 \mathrm{G}$ does not decrease the number of AQP8 molecules in the canalicular membrane in acute low-dose studies in contrast to the reduced numbers that occur with chronic administration. Nevertheless, the possibility exists that the intrinsic water conductance of AQP8 may be modulated or impaired by perturbations in the canalicular membrane related to the binding of $\mathrm{E}_{2} 17 \mathrm{G}$ to MRP2/ABCC2. OA, organic anion.
To our knowledge, no published studies exist to demonstrate the effect of deleting Aquaporin- 8 on bile flow. Studies not yet published ( $\mathrm{T}$. Ma, personal communication) report a severe reduction in bile flow. A published study of the physiologic effects in an Aquaporin-8 null mouse focused on fat absorption, which was minimally affected (Yang et al., 2005); the authors thus concluded that there was little effect on bile acid excretion. Although the conclusion is reasonable, it does not relate to an effect on canalicular water flow. The findings in the Claudin-2 null mouse indicate that a 50\% reduction in water flow can occur with no change in bile acid output. Thus, monitoring only bile acid output in the Aquaporin- 8 null mouse does not provide information concerning changes that may have occurred in bile flow.

Canalicular Aquaporin-8 expression is increased in choleresis induced by glucagon (Gradilone et al., 2003; Soria et al., 2009) and endothelin 1 and 3 (Rodriguez et al., 2013) via mechanisms involving trafficking and insertion of Aquaporin8-containing vesicles as well as by modifying its gene expression. Decreased canalicular Aquaporin-8 expression has been observed in cholestatic liver disease associated not only with estrogen, as mentioned below, but also with endotoxin (Lehmann et al., 2008), sepsis (Lehmann and Marinelli, 2009), and extrahepatic biliary obstruction (Carreras et al., 2003). An increase in Aquaporin-8 expression has also been reported to be the physiologic basis for the $35 \%$ increase in bile flow that occurs in the hypoxia-inducible factor knockout mouse (Asai et al., 2017). Thus, all of these findings, together with the sustained effects of $\mathrm{E}_{2} 17 \mathrm{G}$ (discussed below), imply an important regulatory role for Aquaporin-8 expression in the pathogenesis of naturally occurring and xenobioticrelated cholestatic events.

In normal or cholestatic hepatocytes, it is unknown whether Aquaporin-8 single water permeability can be modified. Nevertheless, recent evidence in nonhepatic cells indicates that Aquaporin-8-dependent water transport can be reduced by reversible inhibition of water channel conductance (Medraño-Fernandez et al., 2016). This may be important in $\mathrm{E}_{2} 17 \mathrm{G}$ cholestasis, in which there is clear evidence for an osmotic gradient-independent inhibition of biliary water secretion (see Fig. 2 and discussion below). The activation of $\mathrm{Ca}^{2+}$-dependent protein kinase $\mathrm{C}$ plays a critical role in $\mathrm{E}_{2}$ 17G-induced cholestasis (Crocenzi et al., 2008). Protein kinase $\mathrm{C}$ can phosphorylate the bile salt export pump (BSEP)/ ABCB11, which would alter its canalicular localization and likely its intrinsic activity (Lam et al., 2010). Diverse Aquaporins, including Aquaporin-8, may undergo protein kinase C phosphorylation (Nesverova and Törnroth-Horsefield, 2019), which causes a decrease in water channel permeability in some cases. Thus, the possibility exists that in $\mathrm{E}_{2} 17 \mathrm{G}$-induced cholestasis, protein kinase $\mathrm{C}$ activation may directly or indirectly impair Aquaporin-8 single water permeability.

$\mathbf{E}_{\mathbf{2}} \mathbf{1 7 G} . \mathrm{E}_{2} 17 \mathrm{G}$ is a naturally occurring hormone metabolite that is used to induce cholestasis in animals because it is known that some women will develop cholestasis when taking ethynyl estradiol and, when not taking an oral contraceptive, will develop cholestasis in pregnancy, usually in the third trimester. However, the latter is an uncommon event that is not known to be related to excessive hormone production, and it is therefore attributed to host-related environmental and/or genetic determinants. A variety of experimental designs have been used to characterize the cholestatic effects of $\mathrm{E}_{2} 17 \mathrm{G}$, 
A

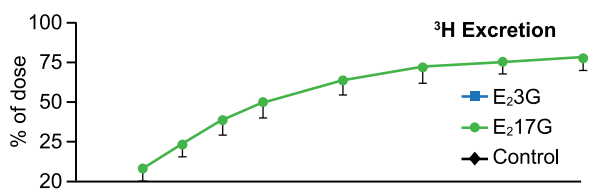

B

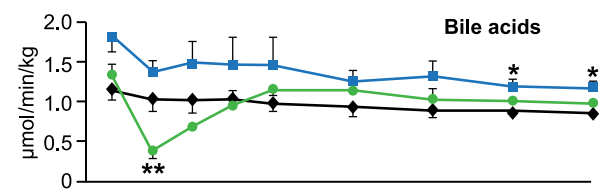

C

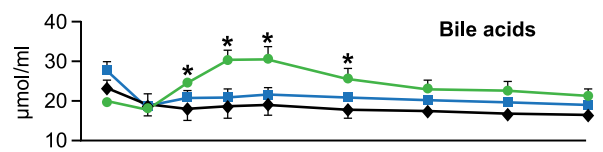

D

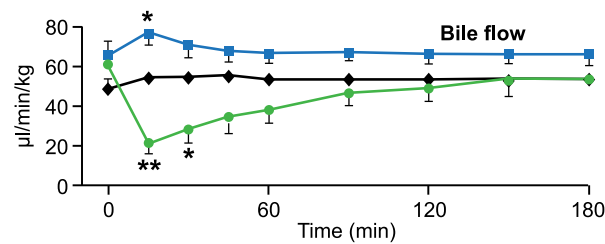

E

Time ( $\mathrm{min})$ $\begin{array}{cccccccccc}\text { Bile acid output } & \mathbf{0 - 1 5} & \mathbf{1 5 - 3 0} & \mathbf{3 0 - 4 5} & \mathbf{4 5 - 6 0} & \mathbf{6 0 - 9 0} & \mathbf{9 0 - 1 2 0} & \text { TOTAL } \\ \text { Control }(\mu \mathrm{mol}) & 16.5 & 15 & 15 & 15 & 30 & 30 & 121.5 \\ & \mathrm{E}_{2} 17 \mathrm{G}(\mu \mathrm{mol}) & 6 & 10.5 & 14.3 & 16.5 & 36 & 36 & 119.3\end{array}$

Volume ( $\mu$ l)

$\begin{array}{llllllll}\text { Control } & 750 & 780 & 780 & 780 & 1560 & 1560 & 6,210 \\ \text { E }_{2} 17 \mathrm{G} & 300 & 345 & 450 & 525 & 1350 & 1470 & 4,440\end{array}$

\% Decrease in Bile acid output $=1.8 \%$ Decrease in Bile Flow $=28.5$

Fig. 2. Effect of a single intravenous dose of ${ }^{3} \mathrm{H}-\mathrm{E}_{2} 17 \mathrm{G}(11 \mu \mathrm{mol} / \mathrm{kg})$ on (A) percent total biliary ${ }^{3} \mathrm{H}$ excretion, (B) biliary bile acid excretion, $\mu \mathrm{mol} / \mathrm{min} / \mathrm{kg}$ body weight (C) bile acid concentration in bile, $\mu \mathrm{mol} / \mathrm{ml}$, (D) bile flow, $\mu \mathrm{l} / \mathrm{min} / \mathrm{kg}$ body weight, and (E) calculated bile acid output and bile flow shown in panels B and D. As shown in (D), bile flow fell to a nadir 15 min after $\mathrm{E}_{2} 17 \mathrm{G}$ administration. However, as shown in (C), no significant change in bile acid concentration occurred until the bile flow recovery phase. As bile flow returned toward the preinjection level, a significant increase in bile acid concentration was found in three successive samples $(15,30$, and 45 minutes $)$. The percent recovery of the injected $\left[{ }^{3} \mathrm{H}\right]$ is shown in (A) and bile acid excretion rates (in micromoles per minute per kilogram body weight) are shown in (B). Data are from Meyers et al. (1980) with permission.

including a range of single, acute doses and infusion of high doses. However, it remains difficult to distinguish physiologic effects of the hormone from pathophysiologic or secondary effects.

The lowest acute dose of $\mathrm{E}_{2} 17 \mathrm{G}$ given to a rat with bile duct drainage and not receiving a bile acid infusion caused an immediate transient $63 \%$ reduction in bile flow that returned to the preinjection level within 90 minutes (Meyers et al., 1980). No change in bile acid concentration occurred in the fluid collected as bile flow decreased; however, a significant increase in bile acid concentration occurred during the recovery of bile flow (Fig. 2).

If the effect of $\mathrm{E}_{2} 17 \mathrm{G}$ was to cause water reabsorption from the biliary tree distal to the canaliculi (i.e., in the bile ducts), then the increase in bile acid concentration should have occurred initially as bile flow diminished. The significant increase in bile acid concentration in the fluid collected during the recovery phase indicates that $\mathrm{E}_{2} 17 \mathrm{G}$ lowered canalicular water flow to a greater extent than carrier-mediated bile acid transport.

Utilizing a different experimental design, the perfused rat liver with a constant infusion of sodium taurocholate, the lowest dose of $\mathrm{E}_{2} 17 \mathrm{G}$ again caused an immediate transient reduction in bile flow (Adinolfi et al., 1984). Neither an increase nor decrease in bile acid concentration was found in collected bile samples, perhaps related to the accumulation of taurocholate in hepatocytes, since the infusion rate exceeded the excretion rate of this bile acid. Nevertheless, both models focus on reduced water flow independent of bile acid concentration. These data are consonant with the experimental model, in which the intact rat is treated with ethynyl estradiol daily for 5 days and basal bile flow is significantly less (Harkavy and Javitt, 1969).

Figure 2, taken from the initial study (Meyers et al., 1980), summarizes the total volume of hepatic bile and total bile acid excretion during the 120-minute period after a low dose of $\mathrm{E}_{2} 17 \mathrm{G}$ (11 $\mu \mathrm{mol} / \mathrm{kg}$, i.v.). Compared with the control, there was a $28.5 \%$ reduction in bile flow and a $2 \%$ reduction in bile acid output, accounting for a significant increase in bile acid concentration.

Studies in hepatocytes and in polarized hepatocyte-derived WIF-B cells (Gradilone et al., 2005; Tietz et al., 2005) suggest that Aquaporin-8, the organic anion transporter multidrug resistance associated protein 2 (MRP2)/ABCC2, and the bile salt transporter BSEP/ABCB11, along with other transporters, are colocalized in the canalicular membrane. Other studies indicate that $\mathrm{E}_{2} 17 \mathrm{G}$ binds to MRP2/ABCC2 and then is rapidly transported into the canaliculus (Gerk et al., 2004). Although the number of Aquaporin-8 channels is not altered in acute studies (Mottino et al., 2006), transient changes in the topography of the membrane as $\mathrm{E}_{2} 17 \mathrm{G}$ is transported via MRP2/ABCC2 (Mottino et al., 2002) into the canaliculus could alter rates of both water flow and solute transport via their respective proteins.

Thus, the data support the view that the acute effect of $\mathrm{E}_{2} 17 \mathrm{G}$ is localized to the canalicular membrane where it binds to and is transported by MRP2/ABCC2. It is quite possible that MRP2/ABCC2-mediated transport of $\mathrm{E}_{2} 17 \mathrm{G}$ can change MRP2/ABCC2 topography in a manner that decreases Aquaporin-8 function. It may now be possible to determine whether $\mathrm{E}_{2} 17 \mathrm{G}$ binds directly to Aquaporin-8 expressed in a lipid membrane and alters its function. Thus, measurement of osmotic membrane water permeability could be assessed after reconstitution of purified Aquaporin- 8 in lipid vesicles pretreated with $\mathrm{E}_{2} 17 \mathrm{G}$ or estradiol-3-glucuronide $\left(\mathrm{E}_{2} 3 \mathrm{G}\right)$. Similarly, the requirement for MRP2/ABCC2 could be assessed by expression of MRP2/ABCC2 together with Aquaporin-8 in lipid vesicles pretreated with $\mathrm{E}_{2} 17 \mathrm{G}$ or $\mathrm{E}_{2} 3 \mathrm{G}$. Characterization of the effects of $\mathrm{E}_{2} 3 \mathrm{G}$, which does not induce cholestasis (Meyers et al., 1980) but is transported by MRP2/ABCC2 (Gerk et al., 2004), on Aquaporin-8 function is needed as a critical control. An effect of $E_{2} 17 G$ on the Claudin-2 protein has not been studied. The elegant studies of Matsumoto et al. (2014) demonstrated that the normal role of Claudin-2 in increasing paracellular water inflow could be adapted to directly assess the effect of $\mathrm{E}_{2} 17 \mathrm{G}$ on tight junction permeability. These authors used isolated mouse intrahepatic bile duct units to measure transepithelial water permeability 
in the presence of hypo- or hyperosmotic buffer. Comparable studies in intrahepatic bile duct units from wild-type mice could thus determine whether transepithelial water permeability is altered in the presence of $\mathrm{E}_{2} 17 \mathrm{G}$ versus $\mathrm{E}_{2} 3 \mathrm{G}$. However, the possibility remains that changes in Claudin-2 water permeability might function to diminish the effect on Aquaporin-8-mediated transmembrane water flow. $\mathrm{E}_{2} 17 \mathrm{G}$ is able to induce the post-translational downregulation of hepatocyte Aquaporin-8 (Carreras et al., 2007). Many other biologic effects of $E_{2} 17 \mathrm{G}$ have been found, such as the increase in permeability of the tight junctions (Mottino et al., 2007), the transient effect on the biliary excretion of taurocholate (Vore et al., 1991), and endocytic internalization of BSEP/ABCB11 (Crocenzi et al., 2003). The molecular basis for the transition from the acute to sustained effects of $\mathrm{E}_{2} 17 \mathrm{G}$ merit further scrutiny with respect to the roles of Aquaporin-8 and Claudin2 in their occurrence.

Bile Acids and Osmotically Determined Flow. Two models now exist in which reductions in canalicular water flow lead to an increase in bile acid concentration in the canaliculi, at least initially, which implies that their carrier-mediated transporter is little affected. The increase in bile acid concentration is at variance with the linear relationship between flow and bile acid excretion that occurred with infusion of sodium taurocholate (Wheeler and Ramos, 1960). Later, it was found that the aggregation number for this bile acid remains relatively constant under varying conditions compared with other bile acids (Small, 1968). Figure 3, adapted from the studies of Carpenter and Lindenbaum (1979), illustrates the decrease in the osmotic coefficient of sodium glycochenodeoxycholate attributable to aggregate formation, which occurs as the concentration of bile acids increases. Thus, the osmotic gradient is proportionally less than the increase in the molar concentration of bile acids. Relatively less water is obligated to flow into the canaliculus.

Adding to the complexity of changes that occur when canalicular bile acid concentration increases is the relationship to carrier-mediated lecithin and cholesterol transport. Ultrastructural studies demonstrate that they appear in the

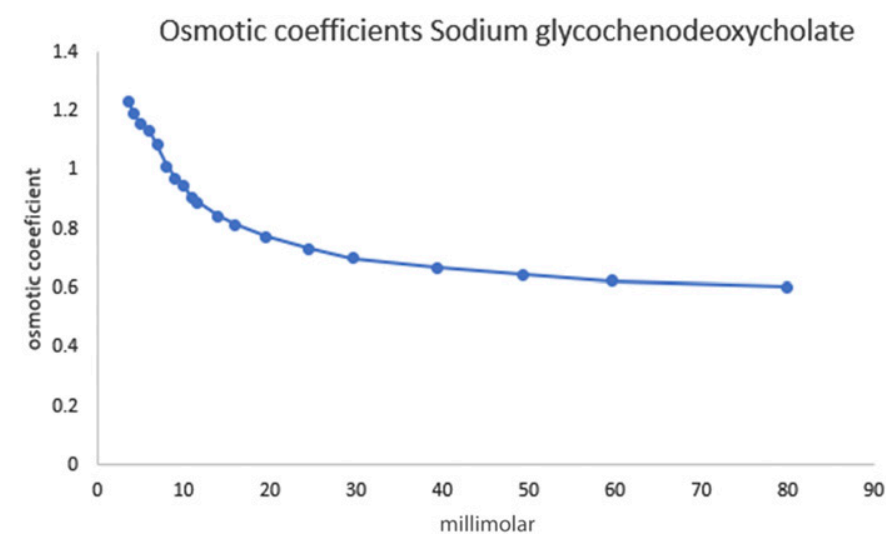

Fig. 3. Osmotic coefficient of sodium glycochenodeoxycholate at 37 degrees C. The osmotic coefficient of sodium glycochenodeoxycholate at different molar concentrations was determined by vapor pressure osmometry (Carpenter and Lindenbaum, 1979). Common to all micelleforming bile acids, the osmotic coefficient decreases with increasing bile acid concentration. Bile acid concentration in bile was $35 \mathrm{mM}$ in the Claudin-2 null mouse compared with $18 \mathrm{mM}$ in the wild-type mouse. Mouse bile is mostly the taurine conjugates of cholic and muricholic acids and would probably have similar changes in their osmotic coefficients. canaliculus as water-insoluble vesicles, which are incorporated into bile acid aggregates to form mixed micelles (Crawford et al., 1995). Their rate of formation is thought to be dependent on the rate of bile acid secretion (Crawford et al., 1995). The transport of lecithin from the canalicular membrane is not sufficient to cause hepatocellular injury. Whether larger doses of $\mathrm{E}_{2} 17 \mathrm{G}$, which lower canalicular water flow by $90 \%$ or more, will cause further increases in bile acid concentrations that extract sufficient amounts of lecithin from the membrane to cause hepatocellular injury has not yet been studied.

\section{Conclusion and Perspectives}

Cholestasis remains a major clinical problem because of its role in progression to cirrhosis and also because it is a hindrance to the development of medications that otherwise would have major beneficial effects. This review focuses on new and old studies that bear on canalicular water flow, the initial site for hepatic bile formation. Although canalicular water flow is dependent on osmotic gradients, it nevertheless is independently regulated by determinants of Aquaporin-8 expression and/or water channel activity, Claudin-2 expression, and probably other determinants that need to be identified. This minireview provides a rationale for evaluating the effects of xenobiotics on hepatic bile formation using acute and chronic administration to wild-type and Claudin-2 or Aquaporin-8 null animals. By differentiating the events occurring in the canaliculus from the more distal regions of the biliary tree, a more precise evaluation is obtained. Knowledge of what happens to the canalicular fluid distal to the canaliculus is also essential to our understanding of hepatic bile formation but in this minireview, we begin at the beginning.

\section{Authorship Contributions}

Wrote or contributed to the writing of the manuscript: Marinelli, Vore, Javitt.

\section{References}

Adinolfi LE, Utili R, Gaeta GB, Abernathy CO, and Zimmerman HJ (1984) Cholestasis induced by estradiol-17 beta-D-glucuronide: mechanisms and prevention by sodium taurocholate. Hepatology 4:30-37.

Asai Y, Yamada T, Tsukita S, Takahashi K, Maekawa M, Honma M, Ikeda M, Murakami K, Munakata Y, Shirai Y, et al. (2017) Activation of the hypoxia inducible factor $1 \alpha$ subunit pathway in steatotic liver contributes to formation of cholesterol gallstones. Gastroenterology 152:1521-1535.e8.

Calamita G, Perret J, and Delporte C (2018) Aquaglyceroporins: drug targets for metabolic diseases? Front Physiol 9:851.

Carpenter PC and Lindenbaum S (1979) Osmotic and activity coefficients of aqueous bile salt solutions at 25, 37, and 45 C. J Solution Chem 8:347-357.

Carreras FI, Gradilone SA, Mazzone A, García F, Huang BQ, Ochoa JE, Tietz PS, Larusso NF, Calamita G, and Marinelli RA (2003) Rat hepatocyte aquaporin-8 water channels are down-regulated in extrahepatic cholestasis. Hepatology 37: 1026-1033.

Carreras FI, Lehmann GL, Ferri D, Tioni MF, Calamita G, and Marinelli RA (2007) Defective hepatocyte aquaporin-8 expression and reduced canalicular membrane water permeability in estrogen-induced cholestasis. Am J Physiol Gastrointest Liver Physiol 292:G905-G912.

Crawford JM, Möckel GM, Crawford AR, Hagen SJ, Hatch VC, Barnes S, Godleski JJ, and Carey MC (1995) Imaging biliary lipid secretion in the rat: ultrastructural evidence for vesiculation of the hepatocyte canalicular membrane. J Lipid Res $\mathbf{3 6}$ : 2147-2163.

Crocenzi FA, Mottino AD, Cao J, Veggi LM, Pozzi EJ, Vore M, Coleman R, and Roma MG (2003) Estradiol-17beta-D-glucuronide induces endocytic internalization of Bsep in rats. Am J Physiol Gastrointest Liver Physiol 285:G449-G459.

Crocenzi FA, Sánchez Pozzi EJ, Ruiz ML, Zucchetti AE, Roma MG, Mottino AD, and Vore $\mathrm{M}(2008) \mathrm{Ca}(2+)$-dependent protein kinase $\mathrm{C}$ isoforms are critical to estradiol 17beta-D-glucuronide-induced cholestasis in the rat. Hepatology 48: 1885-1895.

Gradilone SA, García F, Huebert RC, Tietz PS, Larocca MC, Kierbel A, Carreras FI, Larusso NF, and Marinelli RA (2003) Glucagon induces the plasma membrane 
insertion of functional aquaporin-8 water channels in isolated rat hepatocytes. Hepatology 37:1435-1441.

Gradilone SA, Tietz PS, Splinter PL, Marinelli RA, and LaRusso NF (2005) Expression and subcellular localization of aquaporin water channels in the polarized hepatocyte cell line, WIF-B. BMC Physiol 5:13.

Gerk PM, Li W, and Vore M (2004) Estradiol 3-glucuronide is transported by the multidrug resistance-associated protein 2 but does not activate the allosteric site bound by estradiol 17-glucuronide. Drug Metab Dispos 32:1139-1145.

Harkavy N and Javitt NB (1969) Effect of ethinyl estradiol on hepatic excretory function of the rat, in Metabolic Effects of Gonadal Hormones and Contraceptive Steroids (Salhanick HA, Kipnis DM, and Van de Wiele RL eds) pp 1-19, Plenum Press, New York.

Horner A and Pohl P (2018) Single-file transport of water through membrane channels. Faraday Discuss 209:9-33.

Huebert RC, Splinter PL, Garcia F, Marinelli RA, and LaRusso NF (2002) Expression and localization of aquaporin water channels in rat hepatocytes. Evidence for a role in canalicular bile secretion. $J$ Biol Chem 277:22710-22717.

Lam P, Soroka CJ, and Boyer JL (2010) The bile salt export pump: clinical and experimental aspects of genetic and acquired cholestatic liver disease. Semin Liver Dis 30:125-133.

Lehmann GL, Carreras FI, Soria LR, Gradilone SA, and Marinelli RA (2008) LPS induces the TNF-alpha-mediated downregulation of rat liver aquaporin-8: role in sepsisassociated cholestasis. Am J Physiol Gastrointest Liver Physiol 294:G567-G575.

Lehmann GL and Marinelli RA (2009) Peritoneal sepsis downregulates liver expression of Aquaporin-8: a water channel involved in bile secretion. Liver Int 29:317-318.

Marinelli RA, Tietz PS, Caride AJ, Huang BQ, and LaRusso NF (2003) Water transporting properties of hepatocyte basolateral and canalicular plasma membrane domains. J Biol Chem 278:43157-43162.

Matsumoto K, Imasato M, Yamazaki Y, Tanaka H, Watanabe M, Eguchi H, Nagano $\mathrm{H}$, Hikita H, Tatsumi T, Takehara T, et al. (2014) Claudin 2 deficiency reduces bile flow and increases susceptibility to cholesterol gallstone disease in mice. Gastroenterology 147:1134-1145.e10.

Medraño-Fernandez I, Bestetti S, Bertolotti M, Bienert GP, Bottino C, Laforenza U, Rubartelli A, and Sitia R (2016) Stress regulates aquaporin-8 permeability to impact cell growth and survival. Antioxid Redox Signal 24:1031-1044.

Meyers M, Slikker W, Pascoe G, and Vore M (1980) Characterization of cholestasis induced by estradiol-17 beta-D-glucuronide in the rat. J Pharmacol Exp Ther $\mathbf{2 1 4}$ $87-93$

Mottino AD, Cao J, Veggi LM, Crocenzi F, Roma MG, and Vore M (2002) Altered localization and activity of canalicular Mrp2 in estradiol-17beta-D-glucuronideinduced cholestasis. Hepatology 35:1409-1419.

Mottino AD, Carreras FI, Gradilone SA, Marinelli RA, and Vore M (2006) Canalicular membrane localization of hepatocyte aquaporin- 8 is preserved in estradiol17beta-D-glucuronide-induced cholestasis. J Hepatol 44:232-233.
Mottino AD, Hoffman T, Crocenzi FA, Sánchez Pozzi EJ, Roma MG, and Vore M (2007) Disruption of function and localization of tight junctional structures and Mrp2 in sustained estradiol-17beta-D-glucuronide-induced cholestasis. Am J Physiol Gastrointest Liver Physiol 293:G391-G402.

Muto S, Hata M, Taniguchi J, Tsuruoka S, Moriwaki K, Saitou M, Furuse K, Sasaki H, Fujimura A, Imai M, et al. (2010) Claudin-2-deficient mice are defective in the leaky and cation-selective paracellular permeability properties of renal proximal tubules. Proc Natl Acad Sci USA 107:8011-8016.

Nesverova V and Törnroth-Horsefield S (2019) Phosphorylation-dependent regulation of mammalian aquaporins. Cells 8:E82.

Rodriguez MR, Soria LR, Ventimiglia MS, Najenson AC, Di María A, Dabas P, Fellet A, Marinelli RA, Vatta MS, and Bianciotti LG (2013) Endothelin-1 and -3 induce choleresis in the rat through ETB receptors coupled to nitric oxide and vagovagal reflexes. Clin Sci (Lond) 125:521-532.

Small DM (1968) Size and structure of bile salt micelles. Adv Chem 184:31-51.

Soria LR, Gradilone SA, Larocca MC, and Marinelli RA (2009) Glucagon induces the gene expression of aquaporin-8 but not that of aquaporin- 9 water channels in the rat hepatocyte. Am $J$ Physiol Regul Integr Comp Physiol 296 R1274-R1281.

Sperber I (1959) Secretion of organic anions in the formation of urine and bile. Pharmacol Rev 11:109-134.

Tanaka H, Tamura A, Suzuki K, and Tsukita S (2017) Site-specific distribution of claudin-based paracellular channels with roles in biological fluid flow and metabolism. Ann N Y Acad Sci 1405:44-52.

Tietz P, Jefferson J, Pagano R, and Larusso NF (2005) Membrane microdomains in hepatocytes: potential target areas for proteins involved in canalicular bile secretion. J Lipid Res 46:1426-1432.

Tsukita S, Tanaka H, and Tamura A (2019) The claudins: from tight junctions to biological systems. Trends Biochem Sci 44:141-152.

Vore M, Durham S, Yeh S, and Ganguly T (1991) Hepatic clearance and biliary secretory rate maximum of taurocholate in the recirculating and single pass isolated perfused rat liver. Effects of the cholestatic agent, estradiol-17 beta(beta-D-glucuronide). Biochem Pharmacol 41:431-437.

Wheeler HO and Ramos OL (1960) Determinants of the flow and composition of bile in the unanesthetized dog during constant infusions of sodium taurocholate. J Clin Invest 39:161-170.

Yang B, Song Y, Zhao D, and Verkman AS (2005) Phenotype analysis of aquaporin-8 null mice. Am J Physiol Cell Physiol 288:C1161-C1170.

Address correspondence to: Dr. Norman B. Javitt, New York University Medical Center, Bellevue Hospital C\&D Bldg., 462 First Ave., New York, NY 10016. E-mail: norman.javitt@nyulangone.org 